\title{
Shifted Generalized Pascal Matrices in the Context of Clifford Algebra-Valued Polynomial Sequences
}

\author{
I. Cação ${ }^{1}$, H. R. Malonek ${ }^{1}$, and G. Tomaz ${ }^{1,2}$ \\ 1 Centro de Investigação e Desenvolvimento em Matemática e Aplicações, ${ }^{\star}$ \\ Universidade de Aveiro, 3810-193 Aveiro, Portugal \\ 2 Unidade de Investigação para o Desenvolvimento do Interior, \\ Instituto Politécnico da Guarda, 6300-559 Guarda, Portugal \\ \{isabel.cacao, hrmalon\}@ua.pt, gtomaz@ipg.pt
}

\begin{abstract}
The paper shows the role of shifted generalized Pascal matrices in a matrix representation of hypercomplex orthogonal Appell systems. It extends results obtained in previous works in the context of Appell sequences whose first term is a real constant to sequences whose initial term is a suitable chosen polynomial of $n$ variables.
\end{abstract}

Keywords: Shifted generalized Pascal matrix · Generalized Appell polynomials $\cdot$ Matrix representation

\section{Introduction}

The role of the so-called creation matrix $H$ defined by

$$
(H)_{i l}=\left\{\begin{array}{ll}
i, & i=l+1 \\
0, & i \neq l+1,
\end{array} \quad i, l=0,1, \ldots, m,\right.
$$

has been highlighted in [2] as the main tool for the matrix representation of Appell polynomial sequences of one real variable and its extension to the representation of Sheffer sequences (cf. [1]). The importance of the creation matrix was also confirmed in [3] where the authors developed the matrix representation of homogeneous Appell polynomials that are solutions of a generalized CauchyRiemann system in Euclidean spaces of arbitrary dimensions. In that work the first term of the considered sequence is a real constant. The consideration of a suitable polynomial in $\mathbb{R}^{n}$ as first term leads to more general Appell polynomials (see [16]). Those polynomials also appear in the approach used to construct Gelfand-Tsetlin bases related to branching techniques ([7], [15]). The construction process gives relevance to polynomials obtained by shifting coefficients of the

\footnotetext{
* This work was supported in part by the Portuguese Foundation for Science and Technology ("FCT-Fundação para a Ciência e Tecnologia"), through CIDMA-Center for Research and Development in Mathematics and Applications, within project UID/MAT/04106/2013.
} 
sequence constructed in [13] and reveals their fundamental importance (cf. [10]). The present paper focuses mostly on the representation of those polynomials and stresses that besides $H$, also the shift matrix defined by

$$
(J)_{i l}= \begin{cases}1, & i=l+1 \\ 0, & i \neq l+1, \quad i, l=0, \ldots, m,\end{cases}
$$

plays a significant role. We notice that the pre-multiplication of $J$ by a matrix $A$ results in a matrix whose entries are those of $A$ shifted downward by one position, with zeros in the first row. Also, the post-multiplication causes in A a left shift by one position, with zeros appearing in the last column.

The paper is organized as follows. In Sect. 2 we recall some notions useful in the sequel, namely basic concepts on Clifford analysis and the definitions of generalized and shifted Pascal matrices. In Sect. 3 a matrix approach to the general orthogonal Appell sequences is proposed and some examples are given. A block matrix representation for general systems of orthogonal Appell polynomials is deduced in Sect. 4. We finalize the paper with some conclusions in Sect. 5.

\section{Basic Notions}

Let $\left\{e_{1}, e_{2}, \ldots, e_{n}\right\}$ be an orthonormal basis of the Euclidean vector space $\mathbb{R}^{n}$ endowed with a non-commutative product according to the multiplication rules

$$
e_{i} e_{j}+e_{j} e_{i}=-2 \delta_{i j}, \quad i, j=1,2, \ldots, n,
$$

where $\delta_{i j}$ is the Kronecker symbol. A basis for the associative $2^{n}$-dimensional real Clifford algebra $\mathcal{C} \ell_{0, n}$ is the set $\left\{e_{A}: A \subseteq\{1, \ldots, n\}\right\}$ with

$$
e_{A}=e_{h_{1}} e_{h_{2}} \ldots e_{h_{r}}, \quad 1 \leq h_{1}<\cdots<h_{r} \leq n, \quad e_{\emptyset}=e_{0}=1 .
$$

In general, the vector space $\mathbb{R}^{n+1}$ is embedded in $\mathcal{C} \ell_{0, n}$ by identifying the element $\left(x_{0}, x_{1}, \ldots, x_{n}\right) \in \mathbb{R}^{n+1}$ with the element

$$
x=x_{0}+\sum_{k=1}^{n} e_{k} x_{k}=x_{0}+\underline{x} \in \mathcal{A}_{n}:=\operatorname{span}_{\mathbb{R}}\left\{1, e_{1}, \ldots, e_{n}\right\} \subset \mathcal{C} \ell_{0, n} .
$$

The conjugate $\bar{x}$ and the norm $|x|$ of $x$ are given by $\bar{x}=x_{0}-\underline{x}$ and $|x|=$ $(x \bar{x})^{1 / 2}=(\bar{x} x)^{1 / 2}=\left(\sum_{k=0}^{n} x_{k}^{2}\right)^{1 / 2}$, respectively.

The generalized Cauchy-Riemann operator in $\mathbb{R}^{n+1}$ is defined by

$$
\bar{\partial}:=\frac{1}{2}\left(\partial_{0}+\partial_{\underline{x}}\right),
$$

with $\partial_{0}:=\frac{\partial}{\partial x_{0}}$ and $\partial_{\underline{x}}:=\sum_{k=1}^{n} e_{k} \frac{\partial}{\partial x_{k}}$. The conjugate generalized CauchyRiemann operator, also called the hypercomplex differential operator, is denoted by

$$
\partial:=\frac{1}{2}\left(\partial_{0}-\partial_{\underline{x}}\right) .
$$


We consider $\mathcal{C} \ell_{0, n}$-valued functions defined in an open subset $\Omega \subseteq \mathbb{R}^{n+1} \cong$ $\mathcal{A}_{n}$, i.e. functions of the form $f(z)=\sum_{A} f_{A}(z) e_{A}$ with $f_{A}(z)$ real valued.

A function $f$ is called left (right) monogenic in $\Omega$ if it is a solution of the differential equation $\bar{\partial} f=0(f \bar{\partial}=0)$. From now on we will only use left monogenic functions which we refer to as monogenic functions (right monogenic functions are treated analogously).

The hypercomplex differentiability as generalization of complex differentiability has to be understood in the following way (see [14]): a function $f$ defined in an open domain $\Omega \subseteq \mathbb{R}^{n+1}$ is hypercomplex differentiable supposing there exists in each point of $\Omega$ a uniquely defined areolar derivative $f^{\prime}$. Then $f$ is real differentiable and $f^{\prime}:=\partial f$. Furthermore, $f$ is hypercomplex differentiable in $\Omega$ if and only if $f$ is monogenic. In addition, the monogenicity of $f$ implies that $f^{\prime}=\partial_{0} f=-\partial_{\underline{x}} f$.

Further on we will consider hypercomplex polynomials orthogonal with respect to the Clifford algebra-valued inner product

$$
(f, g)_{\mathcal{C} \ell_{0, n}}=\int_{B^{n+1}} \bar{f} g \mathrm{~d} \lambda^{n+1},
$$

where $\lambda^{n+1}$ is the Lebesgue measure in $\mathbb{R}^{n+1}$ and $\bar{f}$ the conjugate of $f \in \mathcal{C} \ell_{0, n}$.

With regard to the use of matrices in the context of this work, we remember that the well-known Pascal matrix $P$, a lower triangular matrix containing the binomial coefficients as its non-zero entries, i.e.,

$$
(P)_{i l}= \begin{cases}\left(\begin{array}{l}
i \\
l
\end{array}\right), & i \geq l \\
0, & i<l, \quad i, l=0, \ldots, m,\end{cases}
$$

is an essential tool in many applications, particularly in matrix theory and combinatorics. Since all Appell polynomials satisfy a binomial theorem (cf. [12]), it becomes clear that the Pascal matrix plays a central role in the matrix representation of such polynomials. In order to achieve a matrix representation of general systems of orthogonal Appell polynomials we introduce the following matrix:

Definition 1. The shifted generalized Pascal matrix $S_{r}(x), r \in \mathbb{N}_{0}$, is defined by

$$
\left(S_{r}(x)\right)_{i l}=\left\{\begin{array}{ll}
\left(\begin{array}{l}
i+r \\
l+r
\end{array}\right) x^{i-l}, & i \geq l \\
0, & i<l,
\end{array} \quad i, l=0, \ldots, m .\right.
$$

It follows that, the matrix (3) can be written as an exponential matrix expressed jointly by the creation matrix $H$ and the shift matrix $J$ :

$$
S_{r}(x)=e^{(H+r J) x} .
$$

Since $S_{0}(1)=e^{H}=P$ is the Pascal matrix of order $m+1$ and $S_{0}(x)=e^{H x}=$ $P(x)$ the generalized Pascal matrix of the same order, Definition 1 includes both special cases (see [5]). But for variable $r, x=0$ and $x=1$, respectively, it is evident that $S_{r}(0)$ and $S_{r}(1)$ coincide with the identity matrix $I_{m+1}$ and the shifted Pascal matrix $S_{r}$ (see [4]). 
Considering the diagonal matrix $\Lambda_{r}$ whose non-zero entries are

$$
\left(\begin{array}{c}
r+l \\
r
\end{array}\right), \quad l=0, \ldots, m
$$

the following relation between $S_{r}(x)$ and $P(x)$ can be easily established (cf. [5]):

$$
\Lambda_{r}^{-1} S_{r}(x) \Lambda_{r}=P(x) .
$$

Such statement shows the similarity relation between $P(x)$ and $S_{r}(x)$ for given values of $m$ and $r$ as the chosen shift parameter. Moreover, since $P^{-1}(x)=$ $P(-x)$ (cf. [11]), from (6) it follows immediately that the inverse of the shifted generalized Pascal matrix is given by

$$
S_{r}^{-1}(x)=S_{r}(-x) .
$$

Remark 1. All the matrices introduced above could be defined as infinite dimensional square matrices in correspondence with infinite sequences of Appell polynomials. However, we restrict ourselves to the representation of vectors of polynomials up to a certain degree and, consequently, we only need to consider square matrices of order $\mathrm{m}+1$.

\section{Matrix Approach to Orthogonal Hypercomplex Appell Sequences}

Appell's sequence definition, derived from the original work of P. Appell [6], has been adapted to the Clifford analysis framework in the following way (see [13]):

Definition 2. A sequence of homogeneous $\mathcal{C} \ell_{0, n}$-valued polynomials $\left\{\phi_{k}^{(n)}(x)\right\}_{k \geq 0}$ in the variable $x \in \mathcal{A}_{n}$ is called an hypercomplex Appell sequence if, for each $k \geq 0$, the following conditions are verified:

(i) $\phi_{k}^{(n)}(x)$ has exact degree $k$;

(ii) $\bar{\partial} \phi_{k}^{(n)}(x)=0$ for all $x \in \mathcal{A}_{n}$;

(iii) $\partial \phi_{k}^{(n)}(x)=k \phi_{k-1}^{(n)}(x), \quad k \geq 1$.

The approach developed in [3] on the matrix representation of hypercomplex Appell sequences shows that, like in the case of real Appell sequences ([2]), the creation matrix $H$ is the main tool for carrying out that representation.

In fact, the $n$-dimensional homogeneous polynomials

$$
\phi_{k}^{(n)}(x)=\sum_{j=0}^{k}\left(\begin{array}{l}
k \\
j
\end{array}\right) c_{j}(n) \underline{x}^{j} x_{0}^{k-j}, \quad k=0,1, \ldots,
$$

whose coefficients $c_{j}(n)$ verify

$$
c_{0}(n) \neq 0, \quad c_{2 k}(n)=c_{2 k-1}(n)=\frac{\left(\frac{1}{2}\right)_{k}}{\left(\frac{n}{2}\right)_{k}} c_{0}(n), \quad n>1 ; k=1,2, \ldots
$$


$\left((.)_{r}\right.$ denotes the Pochhammer symbol defined by $\left.(a)_{r}=\frac{\Gamma(a+r)}{\Gamma(a)}, r \geq 0\right)$, fulfil all the conditions of the Definition 2, i.e., they form a hypercomplex Appell sequence.

Introducing the vector $\phi^{(n)}(x)=\left[\begin{array}{llll}\phi_{0}^{(n)}(x) & \phi_{1}^{(n)}(x) & \cdots & \phi_{m}^{(n)}(x)\end{array}\right]^{T}$, as consequence of the condition (iii) of the referred definition, we obtain the differential equation

$$
\partial_{0} \phi^{(n)}(x)=H \phi^{(n)}(x)
$$

which together with the vector of initial values on the hyperplane $x_{0}=0$, $\phi^{(n)}(0, \underline{x})=\left[\begin{array}{lll}c_{0} \underline{x}^{0} & c_{1} \underline{x} \cdots c_{m} \underline{x}^{m}\end{array}\right]^{T}$, has the solution

$$
\begin{aligned}
\phi^{(n)}(x) & =e^{H x_{0}} \phi^{(n)}(0, \underline{x}) \\
& =P\left(x_{0}\right) \phi^{(n)}(0, \underline{x}) .
\end{aligned}
$$

Furthermore, introducing the diagonal matrix

$$
D_{c(n)}=\operatorname{diag}\left[c_{0}(n) c_{1}(n) \cdots c_{m}(n)\right],
$$

and the vector $\xi(\underline{x})=\left[1 \underline{x} \cdots \underline{x}^{m}\right]^{T}$, the vector of Appell polynomials (10) becomes

$$
\phi^{(n)}(x)=e^{H x_{0}} D_{c(n)} \xi(\underline{x}) .
$$

Extensions of hypercomplex Appell polynomial sequences can be achieved by considering their first terms, not only a real or complex constant, but as an arbitrary chosen monogenic polynomial $Q_{j}(\underline{x})$, of a fixed degree $j=0,1, \ldots$. Since these polynomials are independent of $x_{0}$, their hypercomplex derivative is the constant zero, i.e., they behave under differentiation like an ordinary constant number. For that reason they are usually called generalized constants or monogenic constants.

With that starting point, the reached sequences include all orthogonal polynomial sequences obtained in the framework of the Gelfand-Tsetlin branching approach $([7],[15])$. The referred orthogonal polynomials are constructed as follows.

Considering, for each integer $k$ and each $j=0, \ldots, k$, the homogeneous polynomial $\tilde{X}_{n+1, j}^{(k)}(x)$ of degree $k$ and index $j$ resulting from the product of a, in general, non-monogenic polynomial of the form

$$
X_{n+1, j}^{(k-j)}(x)=\sum_{s=0}^{k-j}\left(\begin{array}{c}
k \\
j+s
\end{array}\right) d_{j, s}(n) \underline{x}^{s} x_{0}^{k-j-s}
$$

of degree $k-j$, by a homogeneous $\mathcal{C} \ell_{0, n}$-valued monogenic polynomial $Q_{j}(\underline{x})$ of degree $j$, we obtain (see [10])

$$
\begin{aligned}
\tilde{X}_{n+1, j}^{(k)}(x) & :=X_{n+1, j}^{(k-j)}(x) Q_{j}(\underline{x}) \\
& =\sum_{s=0}^{k-j}\left(\begin{array}{c}
k \\
j+s
\end{array}\right) d_{j, s}(n) \underline{x}^{s} x_{0}^{k-j-s} Q_{j}(\underline{x}), \quad x \in \mathcal{A}_{n},
\end{aligned}
$$


where $d_{j, s}(n)$ are real constants.

The monogenicity of $\tilde{X}_{n+1, j}^{(k)}(x)$ depends on the values of the coefficients $d_{j, s}(n)$. In order to obtain such values, we use the following Lemma.

Lemma 1. For each fixed $j(j=0,1, \ldots)$, let $Q_{j}(\underline{x})$ be a homogeneous $\mathcal{C} \ell_{0, n^{-}}$ valued monogenic polynomial of degree $j$, defined in $\mathbb{R}^{n}$. Then

$$
\partial_{\underline{x}}\left(\underline{x}^{s} Q_{j}(\underline{x})\right)=\left\{\begin{array}{l}
-s \underline{x}^{s-1} Q_{j}(\underline{x}), \quad \text { s even } \\
-(n+2 j+s-1) \underline{x}^{s-1} Q_{j}(\underline{x}), \quad s \text { odd }, \quad s=0,1,2, \ldots
\end{array}\right.
$$

Proof. For $s=0$ the result is immediate due to the monogenicity of $Q_{j}(\underline{x})$. For $s=1$, by applying the generalized Leibniz rule ([8]) to the product $\underline{x} Q_{j}(\underline{x})$ with respect to the operator $\partial$, one has

$$
-\partial_{\underline{x}}\left(\underline{x} Q_{j}(\underline{x})\right)=n Q_{j}(\underline{x})+2 \mathbb{E}_{n} Q_{j}(\underline{x})=(n+2 j) Q_{j}(\underline{x}),
$$

where $\mathbb{E}_{n}=\sum_{k=1}^{n} x_{k} \frac{\partial}{\partial x_{k}}$ is the Euler operator in $\mathbb{R}^{n}$. Repeatedly applying the mentioned rule, the result follows by induction on $s$.

Theorem 1. A polynomial of the form (14) is monogenic if and only if the coefficients $d_{j, s}(n)$ verify the following relations:

$$
d_{j, s}(n)= \begin{cases}\frac{j+s}{n+2 j+s-1} d_{j, s-1}(n), & s \text { odd } \\ \frac{j+s}{s} d_{j, s-1}(n), & s \text { even },\end{cases}
$$

with $d_{j, 0}(n) \neq 0$.

Proof. Making use of the Lemma 1, the action of the operator $\bar{\partial}=\partial_{0}+\partial_{\underline{x}}$ on $\tilde{X}_{n+1, j}^{(k)}(x)$ results in

$$
\begin{aligned}
\bar{\partial} \tilde{X}_{n+1, j}^{(k)}(x)=\sum_{s=0}^{k-j-1}\left(\begin{array}{c}
k \\
j+s
\end{array}\right) d_{j, s}(n)(k-j-s) x_{0}^{k-j-s-1} \underline{x}^{s} Q_{j}(\underline{x}) \\
-\sum_{s=1}^{\left\lfloor\frac{k-j}{2}\right\rfloor}\left(\begin{array}{c}
k \\
j+2 s
\end{array}\right) d_{j, 2 s}(n) x_{0}^{k-j-2 s}(2 s) \underline{x}^{2 s-1} Q_{j}(\underline{x}) \\
-\sum_{s=0}^{\left\lfloor\frac{k-j-1}{2}\right\rfloor}\left(\begin{array}{c}
k \\
j+2 s+1
\end{array}\right) d_{j, 2 s+1}(n) x_{0}^{k-j-2 s-1}(n+2 j+2 s) \underline{x}^{2 s} Q_{j}(\underline{x}),
\end{aligned}
$$

where $\lfloor$.$\rfloor is the floor function.$ 
Splitting the odd and the even powers of $\underline{x}$ on the first sum, and gathering the same powers of $x_{0}$ and $\underline{x}, \bar{\partial} \tilde{X}_{n+1, j}^{(k)}(x)$ becomes

$$
\begin{aligned}
\bar{\partial} \tilde{X}_{n+1, j}^{(k)}(x)= & \sum_{s=0}^{\left\lfloor\frac{k-j-1}{2}\right\rfloor}\left[\left(\begin{array}{c}
k \\
j+2 s
\end{array}\right)(k-j-2 s) d_{j, 2 s}(n)\right. \\
& \left.-\left(\begin{array}{c}
k \\
j+2 s+1
\end{array}\right)(n+2 j+2 s) d_{j, 2 s+1}(n)\right] x_{0}^{k-j-2 s-1} \underline{x}^{2 s} Q_{j}(\underline{x}) \\
& +\sum_{s=0}^{\left\lfloor\frac{k-j}{2}-1\right\rfloor}\left[\left(\begin{array}{c}
k \\
j+2 s+1
\end{array}\right)(k-j-2 s-1) d_{j, 2 s+1}(n)\right. \\
& \left.-\left(\begin{array}{c}
k \\
j+2 s+2
\end{array}\right)(2 s+2) d_{j, 2 s+2}(n)\right] x_{0}^{k-j-2 s-2} \underline{x}^{2 s+1} Q_{j}(\underline{x}) .
\end{aligned}
$$

The polynomial $\tilde{X}_{n+1, j}^{(k)}(x)$ is monogenic if and only if $\bar{\partial} \tilde{X}_{n+1, j}^{(k)}(x)=0$, i.e,

$$
\left(\begin{array}{c}
k \\
j+2 s
\end{array}\right)(k-j-2 s) d_{j, 2 s}(n)-\left(\begin{array}{c}
k \\
j+2 s+1
\end{array}\right)(n+2 j+2 s) d_{j, 2 s+1}(n)=0
$$

and

$$
\left(\begin{array}{c}
k \\
j+2 s+1
\end{array}\right)(k-j-2 s-1) d_{j, 2 s+1}(n)-\left(\begin{array}{c}
k \\
j+2 s+2
\end{array}\right)(2 s+2) d_{j, 2 s+2}(n)=0 .
$$

Simplifying (16)-(17) we get, respectively,

$$
(j+2 s+1) d_{j, 2 s}(n)=(n+2 j+2 s) d_{j, 2 s+1}(n),
$$

and

$$
(j+2 s+2) d_{j, 2 s+1}(n)=(2 s+2) d_{j, 2 s+2}(n),
$$

and the result follows.

Notice that, from (15) we can also obtain the recurrence relations, for each fixed $j \geq 0$ :

$$
\begin{aligned}
d_{j, 2 s}(n) & =\frac{(j+2 s) !(n+2 j-2) ! !}{j !(2 s) ! !(n+2 j+2 s-2) ! !} d_{j, 0}(n) \\
d_{j, 2 s-1}(n) & =\frac{(j+2 s-1) !(n+2 j-2) ! !}{j !(2 s-2) ! !(n+2 j+2 s-2) ! !} d_{j, 0}(n), \quad s=1,2, \ldots .
\end{aligned}
$$

Corollary 1. For each fixed $j \in \mathbb{N}_{0}$, the sequence $\left\{\tilde{X}_{n+1, j}^{(k)}(x)\right\}_{k \geq j}$ formed by the polynomials (14) whose coefficients verify (15) is an Appell sequence.

Proof. From the guarantee of monogenecity of the polynomials (14) given by the Theorem 1 , and by applying $\partial_{0}$ to those polynomials it follows,

$$
\begin{aligned}
& \partial_{0} \tilde{X}_{n+1, j}^{(j)}(x)=0, \\
& \partial_{0} \tilde{X}_{n+1, j}^{(k)}(x)=k \tilde{X}_{n+1, j}^{(k-1)}(x), \quad k>j,
\end{aligned}
$$

and the proof is complete. 
The property mentioned in the previous corollary was already used in [9] and $[10]$.

Remark 2. The system $\left\{\tilde{X}_{n+1, j}^{(k)}(x) ; j=0, \ldots, k\right\}_{k \geq 0}$, besides being formed by Appell polynomials, is orthogonal with respect to the inner product (2) (see [9] and [10], for details).

According to the previous corollary, the sequence $\left\{\tilde{X}_{n+1, j}^{(r+j)}(x)\right\}_{r \geq 0} \equiv\left\{\tilde{X}_{n+1, j}^{(k)}(x)\right\}_{k \geq j}$ verifies the Appell property

$$
\partial_{0} \tilde{X}_{n+1, j}^{(r+j)}(x)= \begin{cases}0, & r=0 \\ (r+j) \tilde{X}_{n+1, j}^{(r+j-1)}(x), & r>0 .\end{cases}
$$

Noting that the initial values of $\tilde{X}_{n+1, j}^{(r+j)}(x)$ on the hyperplane $x_{0}=0$ are

$$
\tilde{X}_{n+1, j}^{(r+j)}(0, \underline{x})=d_{j, r}(n) \underline{x}^{r} Q_{j}(\underline{x}), \quad r \geq 0
$$

and denoting the vector

$$
\tilde{\boldsymbol{X}}_{j}(x)=\left[\begin{array}{ll}
\tilde{X}_{n+1, j}^{(j)}(x) & \tilde{X}_{n+1, j}^{(j+1)}(x) \cdots \tilde{X}_{n+1, j}^{(j+m)}(x)
\end{array}\right]^{T},
$$

the corresponding vector of the initial values is

$$
\tilde{\boldsymbol{X}}_{j}(0, \underline{x})=\mathcal{D}_{j} \xi_{j}(\underline{x})
$$

where

$$
\mathcal{D}_{j}=\operatorname{diag}\left[d_{j, 0}(n) d_{j, 1}(n) \cdots d_{j, m}(n)\right]
$$

and

$$
\xi_{j}(\underline{x})=\left[Q_{j}(\underline{x}) \underline{x} Q_{j}(\underline{x}) \cdots \underline{x}^{m} Q_{j}(\underline{x})\right]^{T} .
$$

Now, we can establish the following result.

Theorem 2. For each fixed $j$, the vector $\tilde{\boldsymbol{X}}_{j}(x)$ can be decomposed in the form

$$
\tilde{\boldsymbol{X}}_{j}(x)=S_{j}\left(x_{0}\right) \mathcal{D}_{j} \xi_{j}(\underline{x}) .
$$

Proof. According to the property $(20), \tilde{\boldsymbol{X}}_{j}(x)$ verifies the vector differential equation

$$
\partial_{0} \tilde{\boldsymbol{X}}_{j}(x)=(H+j J) \tilde{\boldsymbol{X}}_{j}(x)
$$

whose general solution is

$$
\tilde{\boldsymbol{X}}_{j}(x)=e^{(H+j J) x_{0}} \tilde{\boldsymbol{X}}_{j}(0, \underline{x}) .
$$

From (4) and (22) the result follows straightforwardly. 
Remark 3. We notice that, the matrix representation of the vector $\boldsymbol{X}_{j}(x)=$ $\left[X_{n+1, j}^{(0)}(x) X_{n+1, j}^{(1)}(x) \cdots X_{n+1, j}^{(m)}(x)\right]^{T}$ of the polynomials (13) is similar to (25); the difference consists in the vector $\xi_{j}(\underline{x})$ which in this case corresponds to $\xi_{0}(\underline{x}) \equiv \xi(\underline{x})=\left[1 \underline{x}^{\cdots} \underline{x}^{m}\right]^{T}$, i.e,

$$
\boldsymbol{X}_{j}(x)=S_{j}\left(x_{0}\right) \mathcal{D}_{j} \xi(\underline{x})
$$

Moreover, when $j=0$, if $d_{0,0}(n)=c_{0}(n)$, it follows that $\mathcal{D}_{0} \equiv D_{c(n)}$ and $\tilde{\boldsymbol{X}}_{0}(x) \equiv \boldsymbol{X}_{0}(x) \equiv \boldsymbol{\phi}^{(n)}(x)$.

Example 1. In order to display an example for $n=2$, we consider the monogenic polynomials $Q_{j}(\underline{x})$ coincident with the polynomials $\zeta^{j}:=\left(x_{1}-e_{1} e_{2} x_{2}\right)^{j}=$ $\left(-e_{1} \underline{x}\right)^{j}$ used in [15] for the construction of an orthogonal basis for the space of homogeneous monogenic polynomials with values in $\mathcal{C} \ell_{0, n}$. In this case, by means of (25) and choosing $d_{j, 0}(n)=1$, we obtain the first polynomials for $j=1$ and $j=2$ :

$$
\begin{aligned}
\tilde{\boldsymbol{X}}_{1}(x) & =S_{1}\left(x_{0}\right) \mathcal{D}_{1} \xi_{1}(\underline{x}) \\
\Leftrightarrow\left[\begin{array}{c}
\tilde{X}_{3,1}^{(1)}(x) \\
\tilde{X}_{3,1}^{(2)}(x) \\
\tilde{X}_{3,1}^{(3)}(x) \\
\tilde{X}_{3,1}^{(4)}(x)
\end{array}\right] & =\left[\begin{array}{cccc}
1 & 0 & 0 & 0 \\
2 x_{0} & 1 & 0 & 0 \\
3 x_{0}^{2} & 3 x_{0} & 1 & 0 \\
4 x_{0}^{3} & 6 x_{0}^{2} & 4 x_{0} & 1
\end{array}\right]\left[\begin{array}{cccc}
1 & 0 & 0 & 0 \\
0 & \frac{1}{2} & 0 & 0 \\
0 & 0 & \frac{3}{4} & 0 \\
0 & 0 & 0 & \frac{1}{2}
\end{array}\right]\left[\begin{array}{c}
\zeta \\
\underline{x} \zeta \\
\underline{x}^{2} \zeta \\
\underline{x}^{3} \zeta
\end{array}\right] \\
& =\left[\begin{array}{c}
\zeta \\
2 x_{0} \zeta+\frac{1}{2} \underline{x} \zeta \\
3 x_{0}^{2} \zeta+\frac{3}{2} x_{0} \underline{x} \zeta+\frac{3}{4} \underline{x}^{2} \zeta \\
4 x_{0}^{3} \zeta+3 x_{0}^{2} \underline{x} \zeta+3 x_{0} \underline{x}^{2} \zeta+\frac{1}{2} \underline{x}^{3} \zeta
\end{array}\right],
\end{aligned}
$$

$$
\begin{aligned}
\tilde{\boldsymbol{X}}_{2}(x) & =S_{2}\left(x_{0}\right) \mathcal{D}_{2} \xi_{2}(\underline{x}) \\
\Leftrightarrow\left[\begin{array}{c}
\tilde{X}_{3,2}^{(2)}(x) \\
\tilde{X}_{3,2}^{(3)}(x) \\
\tilde{X}_{3,2}^{(4)}(x) \\
\tilde{X}_{3,2}^{(5)}(x)
\end{array}\right] & =\left[\begin{array}{cccc}
1 & 0 & 0 & 0 \\
3 x_{0} & 1 & 0 & 0 \\
6 x_{0}^{2} & 4 x_{0} & 1 & 0 \\
10 x_{0}^{3} & 10 x_{0}^{2} & 5 x_{0} & 1
\end{array}\right]\left[\begin{array}{cccc}
1 & 0 & 0 & 0 \\
0 & \frac{1}{2} & 0 & 0 \\
0 & 0 & 1 & 0 \\
0 & 0 & 0 & \frac{5}{8}
\end{array}\right]\left[\begin{array}{c}
\zeta^{2} \\
\underline{x} \zeta^{2} \\
\underline{x}^{2} \zeta^{2} \\
\underline{x}^{3} \zeta^{2}
\end{array}\right] \\
& =\left[\begin{array}{c}
\zeta^{2} \\
3 x_{0} \zeta^{2}+\frac{1}{2} \underline{x} \zeta^{2} \\
6 x_{0}^{2} \zeta^{2}+2 x_{0} \underline{x} \zeta^{2}+\underline{x}^{2} \zeta^{2} \\
10 x_{0}^{3} \zeta^{2}+5 x_{0}^{2} \underline{x} \zeta^{2}+5 x_{0} \underline{x}^{2} \zeta^{2}+\frac{5}{8} \underline{x}^{3} \zeta^{2}
\end{array}\right] .
\end{aligned}
$$

Theorem 3. Let $\phi^{(n+2 j)}(x)=\left[\phi_{0}^{(n+2 j)}(x) \phi_{1}^{(n+2 j)}(x) \cdots \phi_{m}^{(n+2 j)}(x)\right]^{T}$ be the vector of polynomials (8) on the parameter $n+2 j$, for each fixed $j$. Then

$$
\boldsymbol{X}_{j}(x)=\Lambda_{j} \boldsymbol{\phi}^{(n+2 j)}(x)
$$


Proof. Rewriting coefficients (9) in the form

$$
c_{2 s}=c_{2 s-1}=\frac{(2 s-1) ! !(n-2) ! !}{(n+2 s-2) ! !} c_{0}(n), \quad s=1,2, \ldots,
$$

and comparing with (18)-(19), we have

$$
d_{j, s}(n)=\left(\begin{array}{c}
j+s \\
j
\end{array}\right) c_{s}(n+2 j), \quad s=0,1, \ldots
$$

By considering the matrix $\Lambda_{j}$ as defined in (5), the diagonal matrix (11) on the parameter $n+2 j$, and (23), the matrix form of that equality is

$$
\mathcal{D}_{j}=\Lambda_{j} D_{c(n+2 j)} .
$$

Then, taking into account (6), (12), and (26) we have

$$
\boldsymbol{X}_{j}(x)=S_{j}\left(x_{0}\right) \mathcal{D}_{j} \xi(\underline{x})=\Lambda_{j} P\left(x_{0}\right) \Lambda_{j}^{-1} \Lambda_{j} D_{c(n+2 j)} \xi(\underline{x})=\Lambda_{j} \phi^{(n+2 j)}(x)
$$

and the proof is complete.

Remark 4. We observe that the result stated in Theorem 3 converts in matrix form the property established in Theorem 3.1 of [10].

Since the matrix $S_{j}\left(x_{0}\right) \mathcal{D}_{j}$ of $(26)$ is non-singular, powers of $\underline{x}$ may be expressed in terms of the polynomials $X_{n+1, j}^{(k-j)}(x)$. More concretely, we get the following theorem.

Theorem 4. Let $\xi(\underline{x})$ the vector of powers of $\underline{x}$. Then

$$
\xi(\underline{x})=\mathcal{D}_{j}^{-1} S_{j}\left(-x_{0}\right) \boldsymbol{X}_{j}(x) .
$$

Proof. Considering (7) together with (26) the proof is immediate.

Noting that the matrix $\mathcal{D}_{j}^{-1} S_{j}\left(-x_{0}\right)$ is defined by

$$
\left(\mathcal{D}_{j}^{-1} S_{j}\left(-x_{0}\right)\right)_{i l}=\left\{\begin{array}{ll}
\left(\begin{array}{l}
i+j \\
l+j
\end{array}\right) \frac{\left(-x_{0}\right)^{i-l}}{d_{j, i}(n)}, & i \geq l \\
0, & i<l,
\end{array} \quad i, l=0, \ldots, m,\right.
$$

(27) is equivalent to

$$
\sum_{l=0}^{r}\left(\begin{array}{c}
r+j \\
l+j
\end{array}\right) \frac{\left(-x_{0}\right)^{r-l}}{d_{j, r}(n)} X_{n+1, j}^{(l)}(x)=\underline{x}^{r}, \quad r=0,1, \ldots, m .
$$

As consequence, the recurrence relation

$$
X_{n+1, j}^{(r)}(x)=d_{j, r}(n) \underline{x}^{r}-\sum_{l=0}^{r-1}\left(\begin{array}{l}
r+j \\
l+j
\end{array}\right)\left(-x_{0}\right)^{r-l} X_{n+1, j}^{(l)}(x), \quad r=0,1, \ldots
$$

for the polynomials (13) is obtained. 


\section{Block Matrix Representation of a Global Recursive Construction Scheme}

Based on the previous section, it is possible to obtain a block matrix representation for the whole orthogonal system $\left\{X_{n+1, j}^{(k-j)}(x) Q_{j}(\underline{x}) ; j=0, \ldots, k\right\}_{k \geq 0}$.

Setting $k=0, \ldots, m$, the truncated system consists of the non-vanishing entries of the lower triangular matrix $\tilde{\mathcal{X}}$ defined by

$$
(\tilde{\mathcal{X}})_{k j}= \begin{cases}X_{n+1, j}^{(k-j)}(x) Q_{j}(\underline{x}), & k \geq j \\ 0, & k<j, \quad k, j=0, \ldots, m .\end{cases}
$$

It means that its $r$-th column , $0 \leq r \leq m$, is

$$
(\tilde{\mathcal{X}})_{r}=J^{r} \tilde{\boldsymbol{X}}_{r}(x)
$$

where $\tilde{\boldsymbol{X}}_{r}(x)=\left[\begin{array}{ll}\tilde{X}_{n+1, r}^{(r)}(x) & \tilde{X}_{n+1, r}^{(r+1)}(x) \cdots \tilde{X}_{n+1, r}^{(r+m)}(x)\end{array}\right]^{T}$ as defined in $(21)$ and

$$
\left(J^{r}\right)_{k j}=\left\{\begin{array}{ll}
1 & k=j+r \\
0, & k \neq j+r,
\end{array} \quad k, j=0, \ldots, m,\right.
$$

are the powers of the shift matrix (1).

Theorem 5. Let $\mathcal{D}=\operatorname{diag}\left[\mathcal{D}_{0} \mathcal{D}_{1} \cdots \mathcal{D}_{m}\right], \quad \mathcal{J}=\left[\begin{array}{lll}J^{0} & J^{1} \cdots J^{m}\end{array}\right]^{T}$, and $\mathcal{E}=$ $\left[\xi_{0}(\underline{x}) \xi_{1}(\underline{x}) \cdots \xi_{m}(\underline{x})\right]^{T}$ be the block matrices where $\mathcal{D}_{r}, \xi_{r}(\underline{x}), J^{r}, r=0, \ldots, m$, are defined by (23), (28), and (24), respectively. Considering the generalized Pascal matrix $P\left(x_{0}\right)$, the matrix $\tilde{\mathcal{X}}$ can be factorized in the form

$$
\tilde{\mathcal{X}}=P\left(x_{0}\right) \mathcal{J}^{T} \mathcal{D} \mathcal{E}
$$

To prove this theorem we need the following lemma.

Lemma 2. For every $r=0, \ldots, m$,

$$
P(x) J^{r}=J^{r} S_{r}(x) .
$$

Proof. Recalling that

$$
(P(x))_{i l}=\left\{\begin{array}{ll}
\left(\begin{array}{l}
i \\
l
\end{array}\right) x^{i-l} & i \geq l \\
0, & i<l,
\end{array} \quad i, l=0, \ldots, m,\right.
$$

and by using (3) and (28), the product of matrices leads to

$$
\left(P(x) J^{r}\right)_{i l}=\left(J^{r} S_{r}(x)\right)_{i l}=\left\{\begin{array}{ll}
\left(\begin{array}{c}
i \\
l+r
\end{array}\right) x^{i-l-r} & i \geq l+r \\
0, & i<l+r,
\end{array} \quad i, l=0, \ldots, m,\right.
$$

which proves the assertion.

Proof (of Theorem 5). The $r$-th column of $P\left(x_{0}\right) \mathcal{J}^{T} \mathcal{D} \mathcal{E}$ is

$$
\left(P\left(x_{0}\right) \mathcal{J}^{T} \mathcal{D} \mathcal{E}\right)_{r}=P\left(x_{0}\right) J^{r} \mathcal{D}_{r} \xi_{r}(\underline{x}) .
$$

By Lemma 2 and (25) follows that

$$
\begin{aligned}
\left(P\left(x_{0}\right) \mathcal{J}^{T} \mathcal{D} \mathcal{E}\right)_{r} & =J^{r} S_{r}\left(x_{0}\right) \mathcal{D}_{r} \xi_{r}(\underline{x}) \\
& =J^{r} \quad \tilde{\boldsymbol{X}}_{r}(x),
\end{aligned}
$$

which coincides with $(\tilde{\mathcal{X}})_{r}$. Thus, the proof is concluded. 


\section{Conclusions}

In [15] orthogonal bases for the space $\mathcal{M}_{k}\left(\mathbb{R}^{n+1}, \mathcal{C} \ell_{0, n}\right)$, of $\mathcal{C} \ell_{0, n}$-valued homogeneous polynomials of degree $k$, were constructed resulting in polynomials

$$
f_{k, \mu}=X_{n+1, k_{n}}^{\left(k-k_{n}\right)} X_{n, k_{n-1}}^{\left(k_{n}-k_{n-1}\right)} \cdots X_{3, k_{2}}^{\left(k_{3}-k_{2}\right)} \zeta^{k_{2}},
$$

where $\zeta=x_{1}-x_{2} e_{1} e_{2}$ and $\mu=\left(k_{n+1}, k_{n}, \ldots, k_{3}, k_{2}\right)$ is an arbitrary sequence of integers such that $k=k_{n+1} \geq k_{n} \geq \cdots \geq k_{3} \geq k_{2} \geq 0$.

We stress that (26) gives a matrix representation for each building block used in the construction of the polynomial basis (see (29)). In the particular case $n=2$, for each $k \geq 0$, the set $\left\{X_{3, j}^{(k-j)}(x) \zeta^{j} ; j=0, \ldots, k\right\}$ is itself an orthogonal basis for the space $\mathcal{M}_{k}\left(\mathbb{R}^{3}, \mathcal{C} \ell_{0,2}\right)$ (cf. [7]). In other words, in terms of the general constructive scheme in the block matrix representation of Theorem 5 , every $k$-th row contains $k+1$ entries which form a basis of the space $\mathcal{M}_{k}\left(\mathbb{R}^{3}, \mathcal{C} \ell_{0,2}\right)$.

Finally we emphasize that in this paper the key point was the role of both creation and shift matrices. In fact, we showed the relevance of those matrices in the representation of general hypercomplex sequences unifying and generalizing the approach already considered in [2] and [3].

\section{References}

1. Aceto, L., Cação, I.: A matrix approach to Sheffer polynomials. J. Math. Anal. Appl. 446, 87-100 (2017)

2. Aceto, L., Malonek, H.R., Tomaz, G.: A unified matrix approach to the representation of Appell polynomials. Integral Transforms Spec. Funct. 26, 426-441 (2015)

3. Aceto, L., Malonek, H. R., Tomaz, G.: Matrix approach to hypercomplex Appell polynomials. Appl. Num. Math., in Press

4. Aceto, L., Trigiante, D.: The matrices of Pascal and other greats. Amer. Math. Monthly 108, 232-245(2001)

5. Aceto, L., Trigiante, D.: Special polynomials as continuous dynamical systems. In: Cialdea, A., Dattoli, G., He, M. X.,Shrivastava, H. M. (eds.) Lecture Notes of Seminario Interdisciplinare di Matematica. vol. 9, pp. 33-40 (2010)

6. Appell, P.: Sur une classe de polynômes. Ann. Sci. École Norm. Sup. 9 (2), 119-144 (1880)

7. Bock, S., Gürlebeck, K., Lávička, R., Souček, V.: Gelfand-Tsetlin bases for spherical monogenics in dimension 3. Rev. Mat. Iberoam. 28 (4), 1165-1192 (2012)

8. Cação, I., Falcão, M. I., Malonek, H. R.: Laguerre derivative and monogenic Laguerre polynomials: an operational approach. Math. Comput. Modelling 53, 10841094 (2011)

9. Cação, I., Falcão, M. I., Malonek, H. R.: A matrix recurrence for systems of Clifford algebra-valued orthogonal polynomials. Adv. Appl. Clifford Algebras 24 (4), 981994 (2014)

10. Cação, I., Falcão, M. I., Malonek, H. R.: Three-Term Recurrence Relations for Systems of Clifford Algebra-Valued Orthogonal Polynomials. Adv. Appl. Clifford Algebras, Published online: 08 September 2015, 15pp..doi:10.1007/s00006-015-0596-z

11. Call, G. S., Velleman, D. J.: Pascal's Matrices. Amer. Math. Monthly 100 (4), 372-376 (1993) 
12. Carlson, B. C.: Polynomials Satisfying a Binomial Theorem. J. Math. Anal. Appl. 32, 543-558 (1970)

13. Falcão, M. I., Malonek, H. R.: Generalized exponentials through Appell sets in $\mathbb{R}^{n+1}$ and Bessel functions. In: T. E. Simos, G. Psihoyios, C. Tsitouras (eds.). AIP Conference Proceedings, vol. 936, pp. 738-741 (2007)

14. Gürlebeck, K., Malonek, H. R. : A hypercomplex derivative of monogenic functions in $\mathbb{R}^{m+1}$ and its applications. Complex Variables 39, 199-228 (1999)

15. Lávička, R.: Complete orthogonal Appell systems for spherical monogenics. Complex Anal. Oper. Theory 6, 477-489 (2012)

16. Peña Peña, D.: Shifted Appell Sequences in Clifford Analysis. Results. Math. 63, 1145-1157 (2013) 